\title{
Does the effectiveness of recanalization of chronic occlusion depend on the location of the obstruction?
}

\author{
Leszek Bryniarski ${ }^{1}$, Łukasz Klima², Sławomir Surowiec², Krzysztof L. Bryniarski ${ }^{3}$ Michał Terlecki², \\ Dariusz Dudek ${ }^{1}$ \\ ${ }^{1} 2^{\text {nd }}$ Department of Cardiology, Jagiellonian University Medical College, Krakow, Poland \\ ${ }^{2}$ First Department of Cardiology, Interventional Electrocardiology and Hypertension, University Hospital, Krakow, Poland \\ ${ }^{3}$ Department of Interventional Cardiology, Jagiellonian University Medical College, John Paul II Hospital, Krakow, Poland
}

Adv Interv Cardiol 2018; 14, 3 (53): 258-262

DOI: https://doi.org/10.5114/aic.2018.78328

\begin{abstract}
A bstract
Introduction: In past studies, it has been questioned whether success of recanalization of chronic total occlusion (CTO) depends on the location of the occlusion - the circumflex artery $(C x)$ was considered as the most difficult to open.

Aim: To determine whether the effectiveness of recanalization of CTO depends on the location of the obstruction.

Material and methods: From January 2011 to January 2016, a single operator dedicated to chronic total occlusions performed in our center 357 procedures on 337 patients.

Results: Among 337 patients included in the study, $83.4 \%$ were male. Mean age was $62.8 \pm 9.3$ years. Most of the patients had hypertension ( $86.4 \%$ ) and hyperlipidemia (99.4\%), and $28.8 \%$ of them had diabetes. The most frequently opened artery was the right coronary artery (RCA; 52.4\%), followed by the left anterior descending artery (LAD; 29.4\%), and last the Cx (18.2\%). The mean J-CTO score was comparable between the three groups. The success rate of recanalization of CTO was similar for all arteries: $84.5 \%$ in the RCA, $81.9 \%$ in the LAD and $89.2 \%$ in the Cx (overall $p=0.437$ ). Neither procedural complications nor adverse events depended on the location of the CTO.

Conclusions: Our study shows the same efficacy of СTO procedures of all epicardial arteries. We did not observe that effectiveness of recanalization of CTO depends on the location of the obstruction.
\end{abstract}

Key words: chronic total occlusion, percutaneous coronary intervention, lesion characteristics.

Su m m a ry

In the past studies it has been questioned whether success of recanalization depends on the occlusion location, and commonly the circumflex artery was considered as the most difficult to open. In our study, we confirmed similar success rate regardless of occluded artery. Importantly, we showed that when performed by experienced operator, these procedures are not associated with increased risk for patients.

\section{Introduction}

In recent years there has been significant progress in chronic total occlusions (CTO) recanalization - often described as the final frontier in interventional cardiology. Constant development of technology and equipment together with progress in skills of dedicated operators has led to substantial improvement in outcomes [1].

In the past studies it has been questioned whether success of recanalization depends on the occlusion location, and commonly the circumflex artery (Cx) was considered as the most difficult to open [2].

\section{Aim}

Thus, in this study we tried to determine whether the effectiveness of recanalization of chronic occlusion depends on the location of the obstruction.

\section{Material and methods}

From January 2011 to January 2016 a single operator dedicated to chronic total occlusions performed in our center 357 procedures on 337 patients. Coronary CTOs were defined as angiographic evidence of total occlusions with thrombolysis in myocardial infarction (TIMI)

\section{Corresponding author:}

Prof. Leszek Bryniarski MD, PhD, Second Department of Cardiology and Cardiovascular Interventions, Jagiellonian University Medical College, 17 Kopernika St, 31-501 Krakow, Poland, phone: +48 1242473 00, e-mail: I_bryniarski@poczta.fm

Received: 12.04.2018, accepted: 24.06.2018. 
flow grade of 0 and estimated durations of at least 3 months. Time of occlusion has been defined according to the Euro CTO Club terminology as certain (occlusion confirmed in previous angiograms) or probable (clinically confirmed with documented myocardial infarction in the area of the occluded artery without any other possible myocardial related arteries) or possible (undated CTO with TIMI flow 0 and stable coronary artery disease within the last 3 months) [3]. A successful procedure was defined as angiographic success (final residual stenosis $<30 \%$ by visual estimation and TIMI flow grade of 3 after CTO recanalization).

Several techniques were used: antegrade parallel wire, antegrade dissection reentry (ADR), retrograde wire crossing, touching wire, CART and reverse CART (Controlled Antegrade and Retrograde sub-intimal Tracking). All of these techniques have been described in detail in previous publications [4-7]. After predilatation drug-eluting stents (DES) were implanted. The J-CTO Score was calculated for all of the CTO lesions [8]. In-hospital major adverse cardiovascular events were defined as death, emergency repeat revascularization at the previously opened artery, emergency coronary artery bypass grafting (CABG), myocardial infarction or stroke. Complications of general and specific CTO included perforation, damage to the proximal part of the artery, dissection or a blood clot in a contralateral vessel. Non-Q-wave MI was defined as myocardial necrosis marker elevation $>3$ times the upper limit of normal. When new pathological $Q$ waves, in addition to enzyme elevation, were observed on the electrocardiogram, the event was defined as a Q-wave MI.

\section{Statistical analysis}

Continuous variables are presented as means with standard deviation (SD) and discrete variables as numbers and percentages. Student's t-test, the Mann-Whitney $U$ test and the $\chi^{2}$ test were used to compare appro- priate variables. All tests were two-sided and a $p$-value $<0.05$ was considered statistically significant.

\section{Results}

The baseline characteristics were comparable between patients with different arteries revascularized (Table I). Diabetes was most frequent in the circumflex (Cx) group, whereas patients in the right coronary artery (RCA) group had the highest prevalence of peripheral artery disease. The most frequently occluded artery was the RCA (52.4\%), then the left anterior descending artery (LAD; 29.4\%) and last the Cx (18.2\%).

We did not observe any difference in duration of the occlusion, but there was a significance difference in length between groups (Table II). The mean J-CTO score was comparable between the three groups. The group with the occluded RCA had the longest procedure as well as fluoroscopy time, but it did not influence radiation exposure. On the contrary, the highest dose of contrast was used in the LAD group. The retrograde technique was most commonly used in RCA occlusions. Occlusions in the RCA tended to be the longest (Table III). The RCA was also the artery with the highest number of previous attempts.

Neither procedural complications nor adverse events depended on the location. One patient had a stroke after RCA PCl, and 1 patient had a procedure-related myocardial infarction after RCA PCl. We observed several perforations: 4 in the RCA, 4 in the LAD and 1 in the $C x$.

The success rate of recanalization of CTO was similar for all arteries: $84.5 \%$ in the RCA, $81.9 \%$ in the LAD and $89.2 \%$ in the Cx (overall $p=0.437$ ).

\section{Discussion}

In our study, we confirmed the safety and efficacy of CTO procedures performed by an experienced operator in a high volume center. Our results are similar to

Table I. Patients' characteristics

\begin{tabular}{|c|c|c|c|c|}
\hline Parameter & $\operatorname{LAD}(n=101)$ & $C x(n=62)$ & $\operatorname{RCA}(n=174)$ & $P$ overall \\
\hline Male, $n(\%)$ & $80(79.2)$ & $54(87.1)$ & $147(84.5)$ & 0.361 \\
\hline Age [years] & $63.1 \pm 9.8$ & $63.2 \pm 9.3$ & $62.5 \pm 9.1$ & 0.834 \\
\hline $\mathrm{BMI}\left[\mathrm{kg} / \mathrm{m}^{2}\right]$ & $29.7 \pm 7.4$ & $28.4 \pm 4.0$ & $29.4 \pm 5.0$ & 0.307 \\
\hline Hypercholesterolemia, $n$ (\%) & $101(100.0)$ & $62(100.0)$ & $172(98.9)$ & 0.390 \\
\hline Hypertension, n (\%) & $85(84.2)$ & $56(90.3)$ & $150(86.2)$ & 0.537 \\
\hline Diabetes, $n(\%)$ & $36(35.6)$ & $22(35.5)$ & $39(22.4)$ & 0.001 \\
\hline Current smoker, $n(\%)$ & $17(16.8)$ & $12(19.4)$ & $36(20.7)$ & 0.582 \\
\hline Peripheral artery disease & $2(2.0)$ & $6(9.7)$ & $20(11.5)$ & 0.020 \\
\hline COPD & $5(5.0)$ & $6(9.7)$ & $13(7.5)$ & 0.506 \\
\hline
\end{tabular}

Values are mean \pm standard deviation (SD) or $n(\%)$. BMl - body mass index, COPD - chronic obstructive pulmonary disease, CX - circumflex coronary artery, $\angle A D$ - left anterior descending artery, RCA - right coronary artery. 
Table II. Chronic total occlusion characteristics

\begin{tabular}{lcccccc} 
Variable & LAD $(n=105)$ & Cx $(n=65)$ & RCA $(n=187)$ & $P$ overall & $P$ Cx vs. LAD & $P$ Cx vs. RCA \\
\hline Occlusion duration [months] & $13 \pm 25$ & $9 \pm 15$ & $16 \pm 34$ & 0.316 & 0.735 & 0.194 \\
\hline Length [mm] & $25.7 \pm 9.6$ & $22.7 \pm 9.7$ & $29.9 \pm 14.3$ & $<0.001$ & 0.273 & 0.257 \\
\hline Mean J-CTO score & $2.4 \pm 1.0$ & $2.4 \pm 1.3$ & $2.3 \pm 1.1$ & 0.622 & 0.595 & 0.015 \\
\hline Procedural time [min] & $64.9 \pm 27.4$ & $55.7 \pm 25.0$ & $76.3 \pm 35.6$ & 0.001 & 0.157 & 0.001 \\
\hline Fluoroscopy time [min] & $24.5 \pm 16.0$ & $20.1 \pm 12.1$ & $30.7 \pm 19.3$ & $<0.001$ & 0.253 & 0.009 \\
\hline Radiation [Gy] & $2.0 \pm 1.4$ & $1.7 \pm 1.5$ & $1.9 \pm 1.8$ & 0.302 & 0.766 & 0.749 \\
\hline Contrast media [ml] & $368 \pm 150$ & $277 \pm 143$ & $351 \pm 162$ & 0.001 & 0.001 & 0.009 \\
\hline Retrograde, $n$ (\%) & $18(17.1)$ & $5(7.7)$ & $56(29.9)$ & $<0.001$ & 0.080 & 0.999 \\
\hline Stents used & $1.4 \pm 0.6$ & $1.2 \pm 0.4$ & $1.9 \pm 0.9$ & $<0.001$ & 0.260 & $<.001$ \\
\hline
\end{tabular}

Values are mean \pm standard deviation (SD) or $n(\%)$. CX - circumflex coronary artery, LAD - left anterior descending artery, RCA - right coronary artery.

Table III. J-CTO score characteristics

\begin{tabular}{|c|c|c|c|c|}
\hline Variable & $\operatorname{LAD}(n=105)$ & $C x(n=65)$ & RCA $(n=187)$ & $P$ overall \\
\hline Category of difficulty: & & & & 0.857 \\
\hline Easy - intermediate & $21(20.0)$ & $15(23.1)$ & $42(22.5)$ & \\
\hline Difficult - very difficult & $84(80.0)$ & $50(76.9)$ & $145(77.5)$ & \\
\hline \multicolumn{5}{|l|}{ J-CTO score variables, $n$ (\%): } \\
\hline Blunt stump & $69(65.7)$ & $35(53.8)$ & $114(61.0)$ & 0.304 \\
\hline Calcification & $37(35.2)$ & $12(18.5)$ & $54(28.9)$ & 0.064 \\
\hline Bending $>45^{\circ}$ & $8(7.6)$ & $43(66.2)$ & $18(9.6)$ & $<0.001$ \\
\hline Occlusion length $>20 \mathrm{~mm}$ & $79(75.2)$ & $41(63.1)$ & $147(78.6)$ & 0.045 \\
\hline Re-try lesion & $44(41.9)$ & $27(41.5)$ & $103(55.1)$ & 0.042 \\
\hline
\end{tabular}

Values are mean \pm standard deviation (SD) or $n(\%)$. CX-circumflex coronary artery, LAD - left anterior descending artery, RCA - right coronary artery.

those from American, Japanese and European registries [9-13]. According to the unpublished data of the Polish Cardiac Society for the years 2009-2012 the success rate in Poland was $63.6 \%$ for the antegrade technique. In our center the success rate in recanalization of CTO was $87.9 \%$ (antegrade technique) and altogether for both techniques (ante- and retrograde) was 88.2\% (135 vessels out of 153 occlusions) [14]. In the past studies the highest prevalence of CTO was in the RCA, followed by the $C X$ and LAD. On contrast, in our study the CTO was located least frequently in the Cx. However, similarly to worldwide data, the RCA had the highest prevalence of CTO.

Data from previous studies suggest a lower success rate in $\mathrm{CTO} \mathrm{PCl}$ of the $\mathrm{Cx}$. The recently published PROGRESS-CTO score included CX as one of the independent predictors of technical failure [15]. Moreover, in a systematic review and meta-analysis Joyal et al. presented data showing a relatively low success rate of $C x$ recanalization [16]. Contrary to those findings, we did not ob- serve any differences in procedural success depending on the location of the CTO. The reported lower success rates for the circumflex CTO (example of successful recanalization of CTO in Cx artery is shown in Figures 1 and 2). target vessel in previous studies are likely related to the increased tortuosity of this vessel and the less frequent presence of "interventional" collaterals [2]. In a recent study Elias et al. showed that in patients presenting with ST-elevation myocardial infarction and concomitant CTO, the Cx was an independent predictor of poorly developed collaterals [17]. Although in our study the J-CTO score for all arteries was similar, occlusions in the $\mathrm{Cx}$ were shorter, which might have influenced our results. Interestingly, these procedures also had the lowest contrast usage and most rarely the retrograde technique was used. One of the reasons for the disparity between past studies and ours may be that patients in the current study were carefully selected for the procedure by the operator, which in consequence could have resulted in a smaller selection bias. More- 

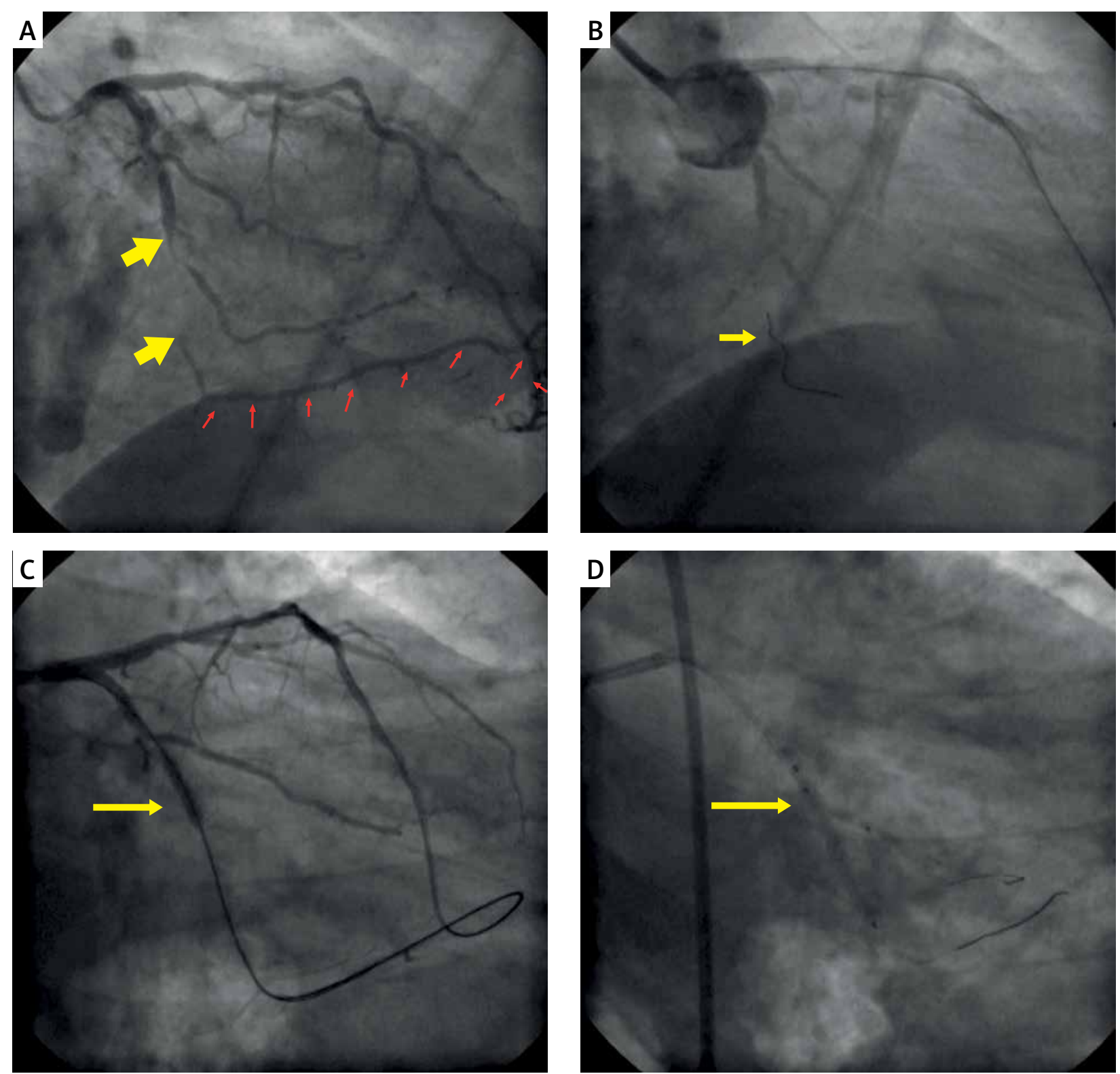

Figure 1. Percutaneous coronary intervention of chronic total occlusion of circumflex artery via ipsilateral approach. A - Occlusion of circumflex coronary artery (yellow arrows); retrograde filling of marginal branch via collaterals from left anterior descending coronary artery (red arrows). B - Retrogradely introduced wire by Corsair microcatheter into the body of the occlusion (arrow). C - Inflated balloon (arrow) introduced antegradely after externalization of retrograde wire. D - Kissing inflation balloons in bifurcation (circumflex coronary artery/marginal branch); at this stage of the procedure both wires were introduced antegradely, and the retrograde system was withdrawn

over, many of them had already undergone failed procedures in other, peripheral centers.

Several limitations of the study should be emphasized. First, post-procedure myocardial necrosis markers were only measured in patients who had post-procedural clinical or ECG signs of MI. Second, although the mean J-CTO was similar in the three arteries, the calcification was less frequent in the $\mathrm{Cx}$ artery and the occluded segment was shorter. This observation could influence the recanalization success rate in the $\mathrm{Cx}$ artery.

\section{Conclusions}

Our observations show the same efficacy of chronic total occlusion procedures of all epicardial arteries. Even though the length of the occlusion in the circumflex was the shortest, the J-СTO score was similar. Moreover, the amount of contrast media, fluoroscopy time and total time of the procedure were the shortest in circumflex artery PCls. Even though statistical significance was not met, it is worth mentioning that the success rate for CTO 


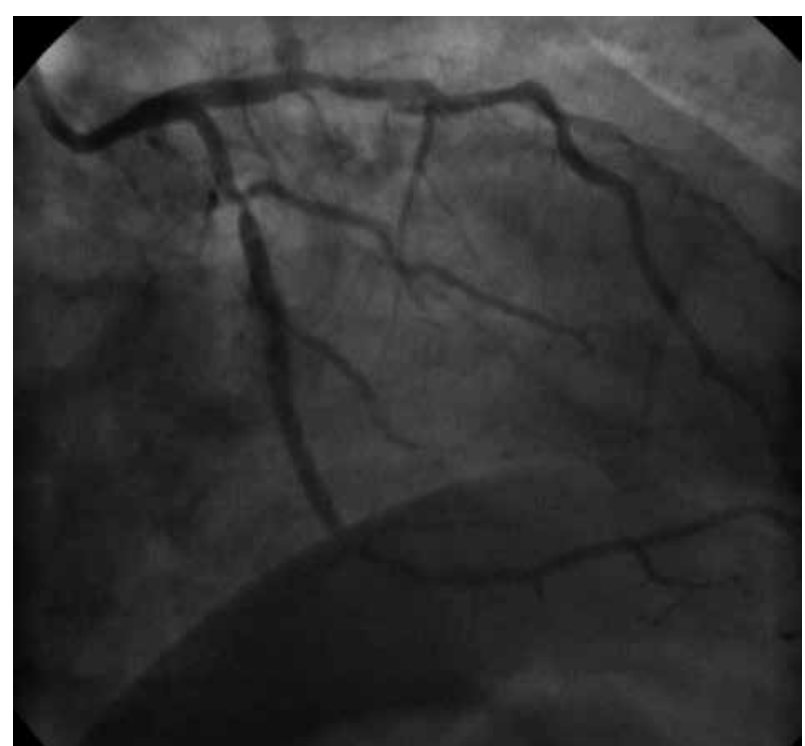

Figure 2. Final result of percutaneous coronary intervention of chronic total occlusion of circumflex artery via ipsilateral approach. Note the preservation of the side branch

Cx was the highest. Our results confirm the high efficacy of chronic total occlusion procedures, and, when performed in experienced centers, these procedures are not associated with increased risk for patients.

\section{Acknowledgments}

Leszek Bryniarski and Łukasz Klima contributed equally.

\section{Conflict of interest}

The authors declare no conflict of interest.

\section{References}

1. Bryniarski L, Bryniarski KL. Which parameters are important before attempting chronic total occlusions recanalization? Adv Interv Cardiol 2016; 12: 96-8.

2. Christopoulos G, Karmpaliotis D, Wyman MR, et al. Percutaneous intervention of circumflex chronic total occlusions is associated with worse procedural outcomes: insights from a Multicentre US Registry. Can J Cardiol 2014; 30: 1588-94.

3. Sianos G, Werner GS, Galassi AR, et al. Recanalisation of chronic total coronary occlusions: 2012 consensus document from the EuroCTO club. EuroIntervention 2012; 8: 139-45.

4. Di Mario C, Werner GS, Sianos G, et al. European perspective in the recanalisation of chronic total occlusions (СTO): consensus document from the EuroCTO Club. Eurolntervention 2007; 3: 30-43.

5. Gülker JE, Bansemir L, Klues HG, Bufe A. Chronic total coronary occlusion recanalization: current techniques and new devices. J Saudi Heart Assoc 2017; 29: 110-5.

6. Muramatsu T, Tsukahara R, Ito Y, et al. Changing strategies of the retrograde approach for chronic total occlusion during the past 7 years. Catheter Cardiovasc Interv 2013; 81: E178-85.

7. Dębski M, Dębski A, Tyczyński P, et al. Retrograde recanalization of chronic total occlusion. A novel maneuver of the old technique. Adv Interv Cardiol 2017; 13: 82-3.
8. Karmpaliotis D, Green P. Chronic Total Occlusion Percutaneous Coronary Intervention in 2014 Beyond the J-CTO Score (Japanese Multicenter CTO Registry): chance favors the prepared mind. JACC Cardiovasc Interv 2015; 8: 268-70.

9. Michael TT, Karmpaliotis D, Brilakis ES, et al. Procedural outcomes of revascularization of chronic total occlusion of native coronary arteries (from a multicenter United States registry). Am J Cardiol 2013; 112: 488-92.

10. Rathore S, Matsuo H, Terashima M, et al. Procedural and in-hospital outcomes after percutaneous coronary intervention for chronic total occlusions of coronary arteries 2002 to 2008: impact of novel guidewire techniques. JACC Cardiovasc Interv 2009; 2: 489-97.

11. Galassi AR, Tomasello SD, Reifart N, et al. In-hospital outcomes of percutaneous coronary intervention in patients with chronic total occlusion: insights from the ERCTO (European Registry of Chronic Total Occlusion) registry. Eurointervention 2011; 7: 472-9.

12. Morino Y, Kimura T, Hayashi Y, et al. In-hospital outcomes of contemporary percutaneous coronary intervention in patients with chronic total occlusion: insights from the J-CTO Registry (Multicenter CTO Registry in Japan). JACC Cardiovasc Interv 2010; 3: 143-51.

13. Baykan AO, Gür M, Acele A, et al. Predictors of successful percutaneous coronary intervention in chronic total coronary occlusions. Adv Interv Cardiol 2016; 12: 17-24.

14. Bryniarski L, Surowiec S, Klima $Ł$, et al. Recanalisation of coronary chronic total occlusion by retrograde approach: the first experience in Poland. Kardiol Pol 2015; 73: 167-76.

15. Christopoulos G, Kandzari DE, Yeh RW, et al. Development and validation of a novel scoring system for predicting technical success of chronic total occlusion percutaneous coronary interventions: the PROGRESS CTO (Prospective Global Registry for the Study of Chronic Total Occlusion Intervention) Score. JACC Cardiovasc Interv 2016; 9: 1-9.

16. Joyal D, Afilalo J, Rinfret S. Effectiveness of recanalization of chronic total occlusions: a systematic review and meta-analysis. Am Heart J 2010; 160: 179-87.

17. Elias J, Hoebers LPC, Dongen IM, et al. Impact of collateral circulation on survival in ST-segment elevation myocardial infarction patients undergoing primary percutaneous coronary intervention with a concomitant chronic total occlusion. JACC CardiovasC Interv 2017; 10: 906-14. 